\title{
Influencia de la educación para el control del fósforo sérico realizada por enfermería a pacientes y a sus cuidadores principales
}

\author{
Laura Hernández Moreno, Romy Judy Perinango, Eva Casanovas Izquierdo
}

InstitutMèdic Badalona (Diaverum Servicios Renales)

\section{Resumen}

Debido a la avanzada edad de la población, esta necesita, cada vez más, la figura de un cuidador principal que controle y se responsabilice de sus cuidados.

Nuestro objetivo es evaluar la efectividad de la educación sanitaria a los pacientes y sus cuidadores principales en la disminución de fósforo sérico.

Se realiza un estudio de 20 meses de duración con una muestra de 70 pacientes divididos en dos grupos; un grupo control que no recibe educación adicional y un grupo estudio que recibe la educación específica para el control del fósforo y su dieta; en este grupo se identifican los pacientes dependientes que requerirán de un cuidador principal, quien también recibirá educación.

Para la identificación del grado de dependencia del paciente se utiliza el Test Delta.

El $52.9 \%$ de nuestros pacientes son independientes; el $48.2 \%$, presentan dependencia leve, y el $4.3 \%$, una dependencia moderada.

El análisis de los resultados pone de manifiesto una disminución significativa del nivel de fósforo sérico en el grupo estudio, consiguiendo que, el $21 \%$ de estos pacientes cuyo nivel de fósforo se encontraba por encima de $5.5 \mathrm{mg} / \mathrm{dl}$, lo redujera hasta los niveles normales recomendados ( $3-5.5 \mathrm{mg} / \mathrm{dl}$ ).

Concluimos que la implicación del personal de enfermería tanto en la educación continua del paciente y de sus cuidadores es necesaria y fundamental para conseguir unos resultados clínicos adecuados.

\section{PALABRAS CLAVE:}

- CUIDADOR PRINCIPAL

- FÓSFORO SÉRICO

- EDUCACIÓN SANITARIA

Correspondencia:

Laura Hernández Moreno

C/Fortuny, 23-27, $2^{\circ} 2^{a}$

08917 Badalona. Barcelona

E-mail: hernandezmorenolaura@gmail.com
Influence of education provided by nurses to patients and their primary caregivers in monitoring serum phosphorus

\begin{abstract}
Due to the advanced age of the population, the figure of the primary caregiver to monitor and take responsibility for their care is most needed.

The aim of study is to evaluate the effectiveness of health education to patients and their primary caregivers in decreasing serum phosphorus.

A study of 20 months duration was carried out with a sample of 70 patients divided into two groups; a control group which received no additional education and a study group which receives specific education for phosphorus control and diet; in the latter group, dependent patients who required a primary caregiver were identified. These primary caregivers also received health education.

Delta Test was used to identify the degree of dependency. Our study found that $52.9 \%$ of our patients were independent; $48.2 \%$ had mild dependence, and $4.3 \%$, moderate dependence.

The analysis of the results revealed a significant decrease in serum phosphorus level in the study group; obtaining that $21 \%$ of these patients whose phosphorus levels were above $5.5 \mathrm{mg} / \mathrm{dl}$ were reduced to the recommended normal levels $(3-5.5 \mathrm{mg} / \mathrm{dl}$ ).

We can conclude that the involvement of nurses in patient education and their primary caregivers is necessary and fundamental to achieve adequate clinical outcomes.
\end{abstract}

\section{KEY WORDS:}

- PRIMARY CAREGIVER

- SERUM PHOSPHORUS

- HEALTH EDUCATION 


\section{Introducción}

En los últimos años la población mayor de 60 años ha aumentado, la gente vive más años y con ello arrastra un serie de patologías propias de su edad, que hace necesario la existencia de un cuidador principal que controle su medicación y/o dieta; entiéndase por cuidador(1) y según el Ministerio de Sanidad, como la persona que asume la responsabilidad en la atención, apoyo y cuidados diarios del enfermo; es quien, además, acompaña la mayor parte del tiempo a esta persona enferma.

En nuestro entorno vemos que la población cada vez es más dependiente de un cuidador principal que suele ser la hija/o o la pareja, y una población adulta que, si bien no depende de un cuidador principal en el control de su medicación, si es dependiente en la preparación de su dieta, entendiéndose como dependencia ${ }^{(2)}$ el estado de carácter permanente en que se encuentran las personas que, por razones derivadas de la edad, de la enfermedad 0 de la discapacidad, y ligadas a la falta 0 pérdida de autonomía física, mental, intelectual o sensorial, precisan de la atención de otra u otras personas, 0 ayudas importantes para realizar actividades básicas de la vida diaria.

\section{Hipótesis}

La educación sanitaria que recibe el paciente en diálisis y su cuidador principal, influye en la disminución de su fósforo sérico.

\section{Objetivo principal}

Evaluar la efectividad de un programa de educación a pacientes y cuidadores en la disminución de su fósforo sérico.

\section{Objetivos específicos}

- Evaluar el nivel de conocimientos que tiene el paciente y su cuidador principal sobre los quelantes de fósforo, así como los alimentos ricos en fósforo.

- Mejorar el grado de información de los pacientes y sus cuidadores principales que están en el programa de diálisis por medio de una intervención educativa dirigida a ellos.

- Elaborar un plan de educación para pacientes en diálisis, y si es necesario, también para su cuidador principal (desde un centro no hospitalario).
- Evaluar la efectividad de la educación sanitaria mediante los parámetros bioquímicos y la respuesta de los pacientes y sus cuidadores a una encuesta posteducación.

\section{Material y método}

Estudio experimental cuantitativo llevado a cabo entre marzo de 2011 y octubre de 2012, cuya muestra está compuesta por 70 pacientes mayores de 18 años que realizan hemodiálisis, sin problemas de disfagia ni obstrucción intestinal o desórdenes severos de la motilidad gastrointestinal, cuyo tratamiento incluye la toma de quelantes de fósforo(3); de los cuales, el $37 \%$ son mujeres y el $63 \%$ hombres.

Se dividen aleatoriamente en dos grupos homogéneos entre sí:

- Grupo A: grupo estudio. Formado por 35 pacientes a los que se revisan y refuerzan sus conocimientos, sobre la importancia de un buen control de los niveles de fósforo sérico ${ }^{(4,5)}$, dieta apropiada y la toma correcta de la medicación ${ }^{(6,7)}$; esta revisión y formación es realizada por enfermería.

- Grupo B: grupo control. Formado por 35 pacientes, quienes no reciben revisión ni refuerzo de sus conocimientos sobre el buen control de los niveles de fósforo sérico, dieta apropiada y la toma correcta de la medicación por parte enfermería.

La media de edad de 68.5 años, a ambos grupos se les realiza, previamente, el test delta ${ }^{(8,9,10)}$ para valorar el nivel de dependencia.

El estudio consta de las siguientes fases:

$1^{\text {a }}$ fase: se inicia en marzo de 2011 , estableciendo los grupos estudio (grupo A) y grupo control (grupo B) recogiendo los datos analíticos de los niveles de fósforo de todos ellos.

$2^{\mathrm{a}}$ fase: comprendida entre los meses de abril y octubre de 2011, se inicia sobre el grupo estudio (A) la revisión de conocimientos y posterior educación sobre qué son los quelantes, cómo actúan, consecuencias de mantener un nivel de fósforo elevado e identificación de los alimentos ricos en fósforo; esta actuación se repite una vez al mes durante los 6 meses que dura esta fase, su duración es de 10 a 15 minutos. Paralelamente, se recogen mensualmente los resultados analíticos de los niveles de fósforo en ambos grupos.

$3^{\text {a }}$ fase: comprendida entre los meses de noviembre de 2011 a noviembre de 2012 , se identifican a los cuidado- 
res principales de los pacientes dependientes pertenecientes al grupo $\mathrm{A}$, a quienes también se revisaran sus conocimientos sobre el fósforo y sus complicaciones; esta fase se subdivide en 3 etapas:

$1^{\text {a }}$ etapa: Contacto telefónico, se les explica el motivo del estudio y su finalidad, se les cita para una entrevista personal pidiéndoles aporten un recordatorio dietético de $48 \mathrm{~h}$ de su familiar.

$2^{\mathrm{a}}$ etapa: Entrevista personal donde se les realiza:

- Encuesta evaluativa de sus conocimientos sobre el control adecuado del fósforo sérico(11,12) con el objetivo de adaptar mejor la revisión de sus conocimientos.

- Se recoge el recordatorio dietético de 48 h para su posterior valoración.

$3^{a}$ etapa: Nueva entrevista para:

- Entrega del recordatorio dietético valorado junto con las recomendaciones oportunas.

- Se realiza test para valorar los conceptos asimilados, si fuera necesario está previsto repetir los refuerzos para asentar seguridad en los conocimientos.

Los datos han sido obtenidos a través del programa de tratamiento de datos específicos del centro, del método estadístico Anova y de las entrevistas personales realizadas a los pacientes y a sus cuidadores principales.

\section{Resultados}

De los 35 pacientes y cuidadores seleccionados para el grupo de estudio, solo un paciente y un cuidador se negaron a participar en el mismo.

Figura 1. Dependencia General

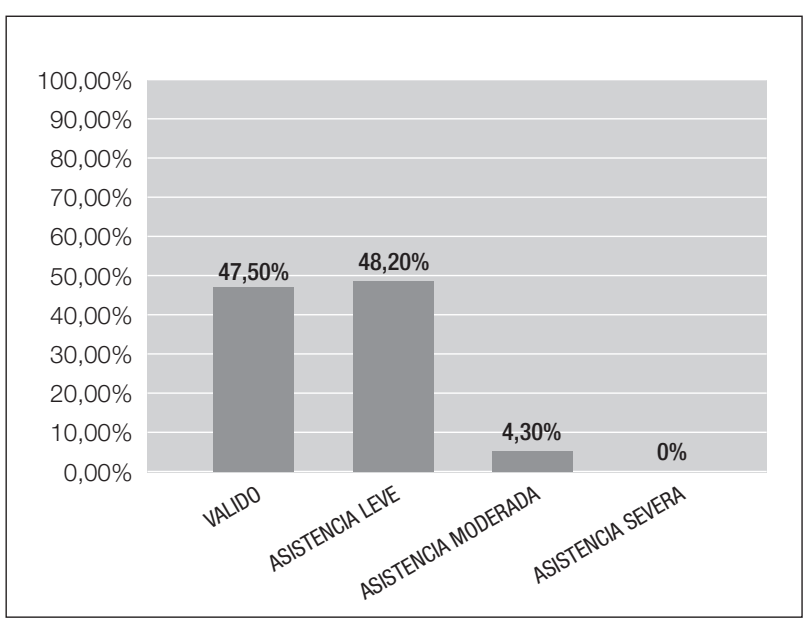

Figura 2. Dependencia Física

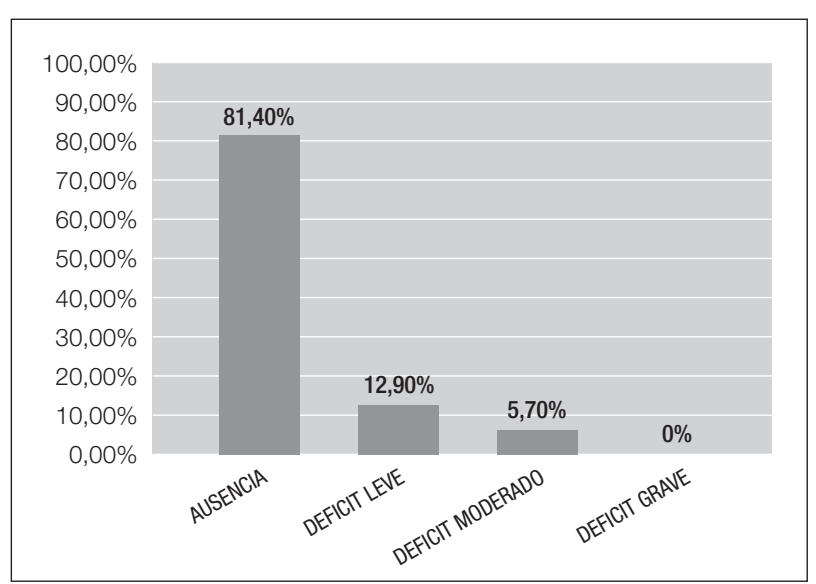

Figura 3. Dependencia Psicológica

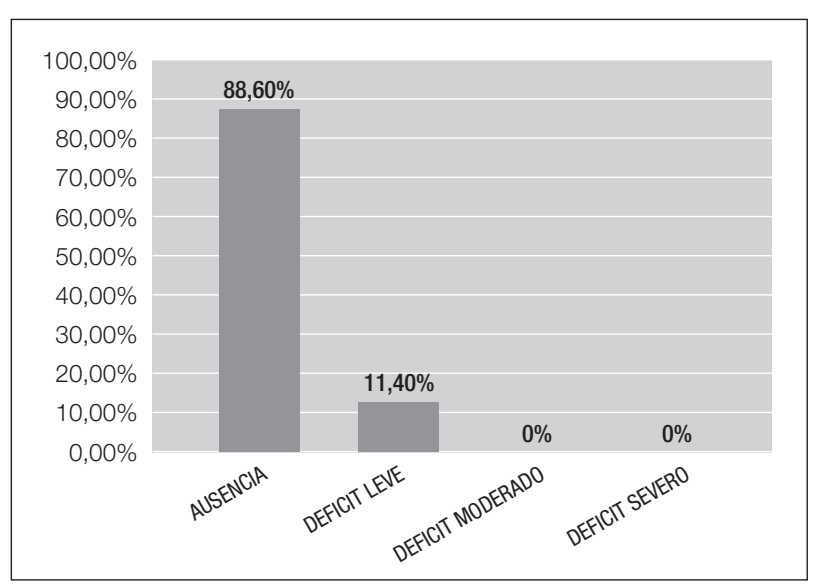

Los resultados del test Delta muestran que el $47,5 \%$ de nuestros pacientes son independientes, el $48,2 \%$ presentan una dependencia leve y el 4,3\%, una dependencia moderada, y que respecto a la dependencia física y la psicológica, obtienen como resultado que el $81,4 \%$ y el $88,6 \%$ no presentan ni dependencia física ni psicológica respectivamente, mientras que el $12,9 \%$ presentan una dependencia física leve y el $11,4 \%$, presentan dependencia psicológica leve; dentro de los pacientes valorados, encontramos que el 5,7\% presentan una dependencia física moderada.

La encuesta inicial refleja que el $50 \%$ los pacientes desconocen la importancia de un buen control de fosforo, el $65 \%$ las complicaciones derivadas, el $90 \%$ no sabe la manera adecuada de controlar correctamente el fosforo, el $50 \%$ no saben identificar de entre la medicación los quelantes del fosforo, el $75 \%$ desconoce la forma correcta de tomarlos y el $70 \%$ no identifican los alimentos más ricos en fosforo (Fig. 4). 
Figura 4. Encuesta inicial conocimientos pacientes

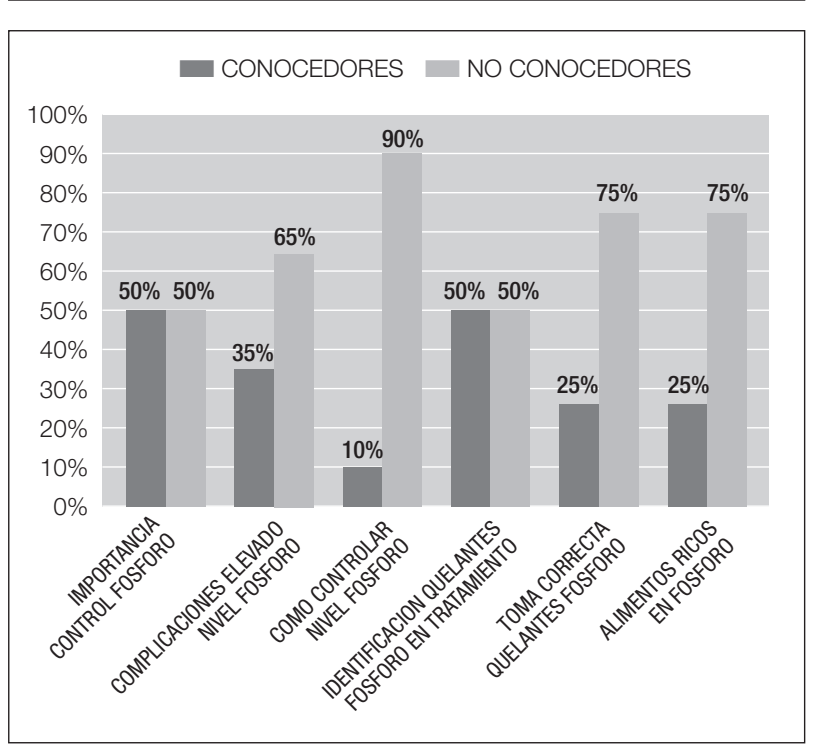

Posterior a la fase educativa, se comprueba que el $100 \%$ de estos pacientes, conocen y explican las complicaciones derivadas de un elevado nivel de fósforo sérico, saben identificar qué alimentos son ricos en fósforo y saben reconocer de entre todos los medicamentos que toman aquellos que son quelantes de fósforo y su correcta forma de administración (Fig. 5).

Figura 5. Encuesta post educación pacientes

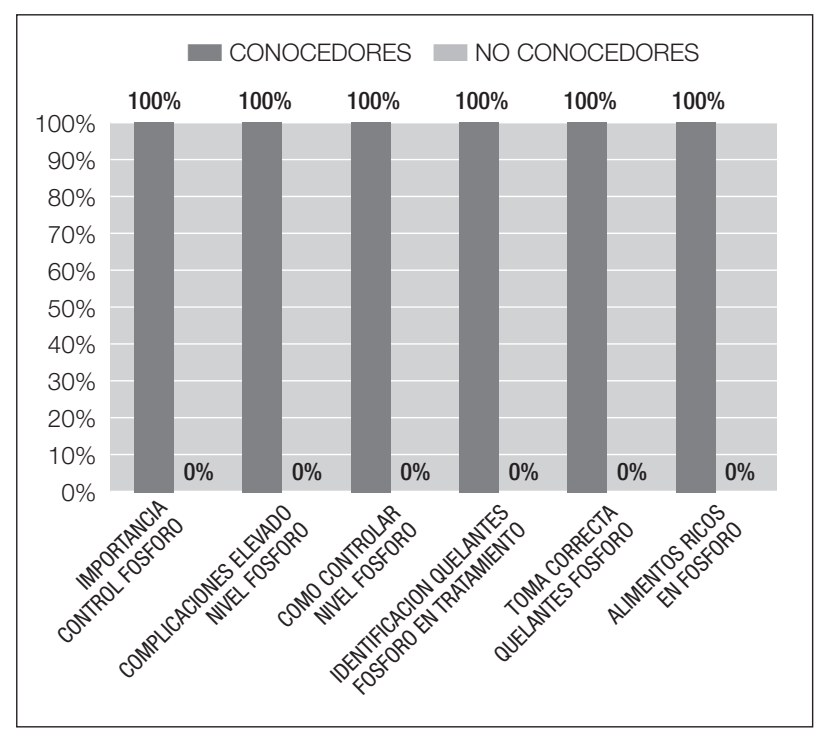

Al inicio de dichas entrevistas, el $50 \%$ los cuidadores desconocen la importancia de un buen control de fosforo, el $80 \%$ las complicaciones derivadas, el $40 \%$ no sabe la manera adecuada de controlar correctamen- te el fosforo, el $70 \%$ no saben identificar de entre la medicación los quelantes fosforo, el $60 \%$ desconoce la forma correcta de tomarlos y el $70 \%$ no identifican los alimentos más ricos del en fosforo (Fig. 6).

Figura 6. Encuesta inicial conocimientos cuidadores

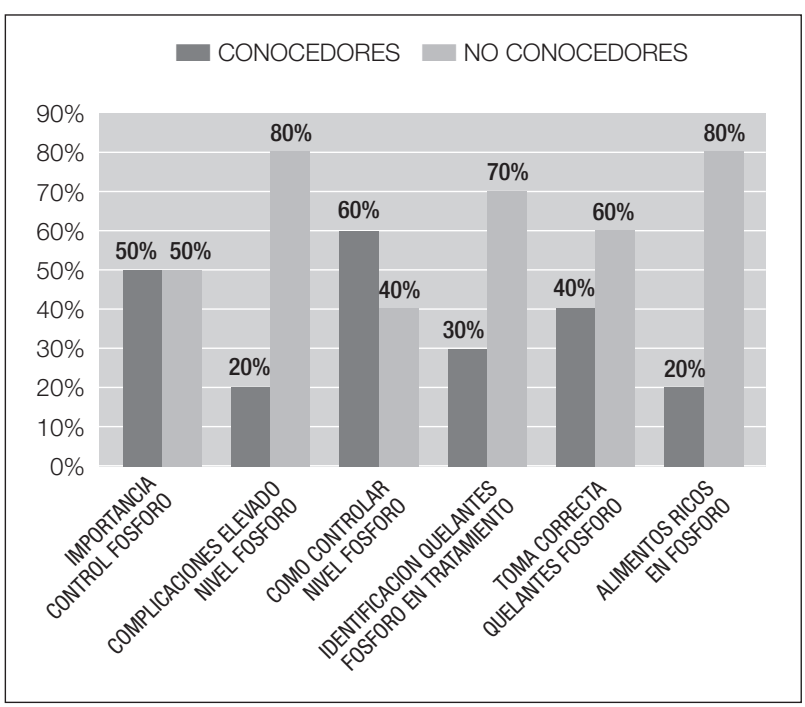

En la valoración final podemos comprobar una evolución positiva en cuanto a conocimientos, ya que el $100 \%$ de estos cuidadores manifiestan conocer las complicaciones derivadas de un elevado nivel de fósforo sérico, saben identificar qué alimentos son ricos en fósforo y saben reconocer de entre todos los medicamentos que toman sus familiares, aquellos que son quelantes de fósforo y su correcta forma de administración ${ }^{(13)}$ (Fig. 7).

Figura 7. Encuesta post educación cuidadores

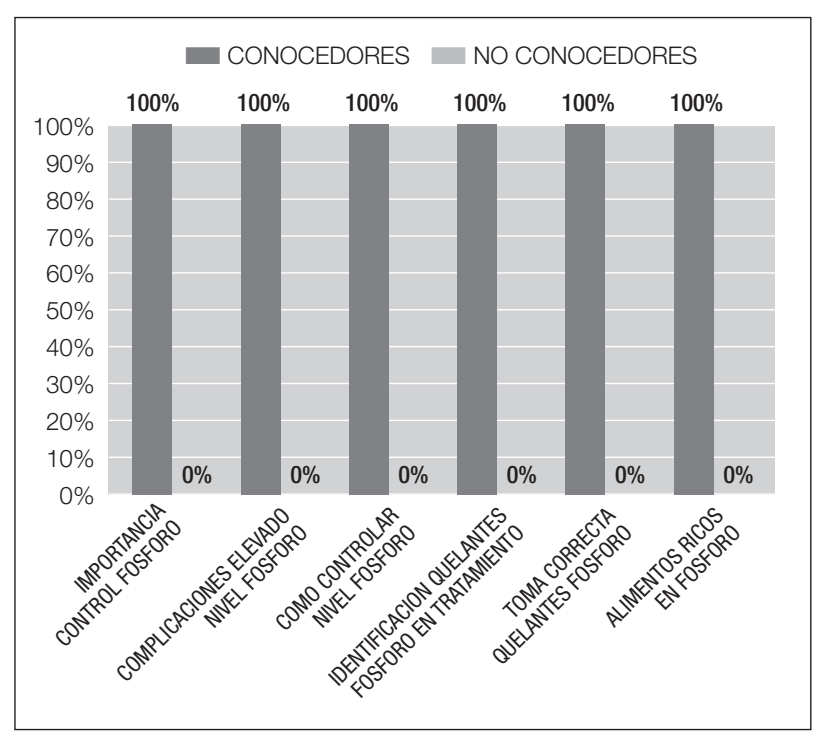


Por otro lado, los resultados analíticos obtenidos mensualmente en ambos grupos A (estudio) y B (control), en el transcurso de los meses, permiten observar la tendencia a la disminución cuantitativa del fósforo (Fig. 8):

Figura 8. Evolución niveles de fósforo sérico

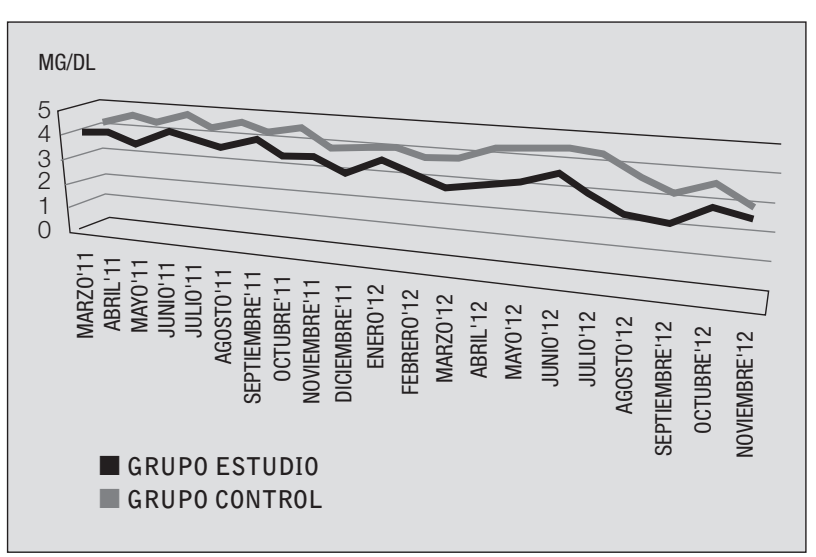

Si reflejamos los valores medios de fosforo sérico obtenidos durante el estudio en tablas podemos observar de forma clara la tendencia en la disminución de los valores. (tabla $\mathbf{1}$ ).

Tabla 1. Media de los niveles de fósforo durante el estudio

\begin{tabular}{|c|c|c|c|}
\hline & Fase 1 & Fase 2 & Fase 3 \\
\hline Grupo estudio & $4.10 \mathrm{mg} / \mathrm{dl}$ & $3.73 \mathrm{mg} / \mathrm{dl}$ & $2.90 \mathrm{mg} / \mathrm{dl}$ \\
\hline Grupo control & $4.10 \mathrm{mg} / \mathrm{dl}$ & $3.88 \mathrm{mg} / \mathrm{dl}$ & $2.92 \mathrm{mg} / \mathrm{dl}$ \\
\hline
\end{tabular}

Como resultados destacables hemos observado la disminución, hasta alcanzar un porcentaje del $0 \%$ de pacientes con valores de fosforo sérico > a 5,5 mg/dl, en grupo estudio ( $A$ ), y que también en la fase final del estudio hay una regularización en los niveles de fosforo sérico en el grupo control (B) con una reducción notable en relación a los valores iniciales.

\section{Discusión}

En la fase final del estudio hemos observado además de la tendencia a la disminución de los niveles de fósforo sérico, oscilaciones en los mismos con un carácter marcadamente estacional especialmente en el grupo estudio, que pensamos pueden ser debidos a la aparición en estos momentos de factores que dificultan el control de la dieta ${ }^{(14)} 0$ cambios de hábitos.

También creemos que ha habido una contaminación positiva por la convivencia, en los tiempos de espera para realizar el tratamiento, con pacientes del grupo (A) hacia los del grupo (B).

Pensamos que, durante la segunda fase del estudio, el grupo (A) presenta valores de fosforo sérico $<3 \mathrm{mg} / \mathrm{dl}$ en un porcentaje elevado, debido al impacto producido por la educación en general y en especial por las imágenes de calcificaciones vasculares sobre vasos importantes en la evolución de la enfermedad y posibles complicaciones que pueden agravar el proceso.

La figura del cuidador principal, es un rol asumido generalmente por las mujeres de la familia, esposa o hijas, siendo este dato semejante al de otros estudios publica$\operatorname{dos}^{(15)}$.

Hemos encontrado pocas referencias que contemplen la figura del cuidador principal(16) por lo que no ha sido posible la comparación de resultados, teniendo en cuenta las características demográficas de la población (edad cada vez más avanzada, grado de dependencia, etc.), creemos importante que la educación ${ }^{(17)}$ enfermera debe ir dirigida también a esa figura para un buen seguimiento y adherencia al tratamiento del paciente. Seguramente en estudios sucesivos, dadas las características de los pacientes incidentes se podrán obtener resultados que permitan mejores comparativas.

Sin embargo, a la vista de nuestros resultados podemos concluir que tras la intervención educativa realizada sobre el paciente y su entorno familiar, hemos conseguido una mejora en la adherencia al tratamiento prescrito, consiguiendo un descenso en los niveles de fósforo sérico de nuesgros pacientes, con los beneficios que ello supone.

Recibido: 17 Diciembre 2013

Revisado: 22 Diciembre 2013

Modificado: 4 Febrero 2014

Aceptado: 20 Febrero 2014

\section{Referencias:}

1. Andreo $L$, Moreno C.: Perfil y realidad social de los cuidadores principales de pacientes dependientes tratados con hemodiálisis. Nursing 2009; 27 (4): 54-65.

2. Camps Ballester $E$, Andreu Periz $L$, Colomer Cordinachs $\mathrm{M}$, ClaramuntFonts $\mathrm{L}$, Pasaron Alonso M.: Valoración del grado de autonomía funcional de pacientes renales crónicos según índices de barthel, lauton y baremo de ley de dependencia. Rev.Enf.Nefrol.2012; 12 (2):104-110 
3. De Francisco A: Captores de fósforo. Nefrología. 2012; 32 (2):240-244.

4. Wilson Astudillo A, Carmen Mendinueta A.: Necesidades de los cuidadores del paciente crónico: guía de recursos sanitarios y sociales en la fase final de la vida en Guipúzcoa. Revista Psicooncología.2008; 5(2): 351-81.

5. Muro Suesrun B, Pernaut Villanueva J, Urzainqui Laborda D.: Control del metabolismo del fósforo en la consulta prediálisis. Rev. Enf. Nefro. 2009; 57(2):1-5.

6. Alonso Latorre M, Blasco Pérez M.J, Castillo Hernández $\mathrm{A}$, Cuevas Cámara $\mathrm{P}$, Febrero $\mathrm{C}$, López $\mathrm{S}$, et al: Influencia de enfermería en el control de la hiperfosfatemia. En: Libro de comunicaciones del XXXII Congreso Nacional SEDEN; 2008. Barcelona Disponible: http://www.revistaseden.org/files/1779_ph 11.pdf.

7. Sánchez I, Lorenzo V.: Hemodiálisis: ¿Cuánto sabemos de los fármacos relacionados con el metabolismo mineral?. Rev.Enferm.Nefrol.2011; 14 (1):23-29.

8. Marco Corredor C, Ruiz Giménez L, Moreno García A, Pérez Ferrer C. Test Delta: Nuestros pacientes, su dependencia. Rev. Enferm Nefrol. 2013; 16 (3):107107.

9. Hernández Arribas V, Palacios García G, Rubio Gil F.J, Porras Montero G, Rodríguez Peña J, Uguet Canal M, Fernández Lucas M.: Evaluación del grado de dependencia de los pacientes en hemodiálisis mediante el test delta. Rev.Enf.Nefro. 2006; 26 (5):600-608.

10. Ochoa Alfaro B.M, González Castillo S, Santesteban de la Concepción A, García Castillejo M.J, Barricarte Navarroe E, Zapatería Azcona C: Análisis del grado de dependencia de pacientes en programa de hemodiálisis periódica en un hospital comarcal. Rev. Enferm.Nefrol. 2009; 8 (3):2-5.
11. González M, Álvarez P, Vázquez J, Rodríguez M, Outeriño S, Manso B, et al: Efectividad de la intervención educativa en cuidadores de pacientes dependientes en diálisis y valoración de la carga. Revista de Nefrología 2005; 8 (2):156-165.

12. Arrojo A, Fernández A, Ruiz M.L, Fernández G, Sancho N.: La intervención enfermera: una garantía en el control de la hiperfosfatemia. Rev.Enferm.Nefrol.2010; 6(2):120-124.

13. Villas $A$, Mañé $N$, Martínez $G$, Masip $M$, López $M$, Picazo L, Ponz E, Real J, Rocabert M, Valenzuela M.P, Yuste E.: Análisis del cumplimiento farmacológico de los pacientes en hemodiálisis.. Rev.Enferm. Nefrol. 2006; 4 (2):147-156.

14. Mayor Iturburuaga J.M.: La alimentación en el enfermo renal, desde el punto de vista de enfermería ¿Cómo reforzar el cumplimiento?. Revista Osasunaz. 2007; 8(2):151-169.

15. Moral Serrano M.S, Ortega Juan, López Matoses M.J, Pellicer Magraner P: Perfil y riesgo de morbilidad psíquica en cuidadores de pacientes ingresados en su domicilio. Rev. Atención Primaria. 2009; 32(1): 77-83.

16. Torres Egea $\mathrm{M}^{\mathrm{a}}$ Pilar, Ballesteros Pérez Esperanza, Sánchez Castillo Pablo David. Programas e intervenciones de apoyo a los cuidadores informales en España. Gerokomos .Madrid. 2008; 19 (14): 25-29.

17. Rota L, Yuste E, Mañé N, García E, Marcet M, Marquina D, Ramírez N.: Diseño de una consulta de enfermería. Educación a los pacientes en hemodiálisis. Rev.Enferm.Nefrol.2002; 7 (3):91-96. 